\title{
CRYOGENIC TESTING OF OPTICS FOR ISOCAM
}

\author{
JOHN K. DAVIES \\ Royal Observatory, Edinburgh, EH9 $3 H J$, Scotland
}

\begin{abstract}
The ROE ISOCAM optical test facility is described. The results of testing of lenses shows good agreement with theory and remarkable consistency.
\end{abstract}

\section{Introduction}

ISOCAM is an infrared camera operating in the 2.5 to 16 micron range which forms part of the payload of the ESA Infrared Space Observatory. The camera has two selectable channels, each with its own optics and detector system. Each channel includes 4 lenses, giving image scales from 1.5 to 12 arcseconds per pixel, interference filters and CVF sets. The lenses, which are of plano-aspheric form, were diamond machined from silicon (short wavelength channel) and germanium (long wavelength channel). After the application of anti-reflection coatings by OCLI and mounting of the lenses by ISOCAM prime contracter Aerospatiale, the individual lenses were tested by the Royal Observatory Edinburgh at $4 \mathrm{~K}$ to verify their performance. Filters were procured from Spectrogon via the Stockholm Observatory and, after mounting by Aerospatiale, tested by ROE to confirm that the required passbands and transmissions were achieved at $4 \mathrm{~K}$.

\section{The ROE Test Facility}

The test facility is designed to provide an environment optically similar to that encountered in ISOCAM. The test cryostat is representative of one channel of the camera and lenses and filters are mounted in their holders, although not in the actual ISOC AM lens/filter wheels. All tests are carried out with the cryostat cooled to $4 \mathrm{~K}$ by a combination of a closed cycle cooler and liquid helium boiloff.

A schematic diagram of the test facility is shown in Figure 1, it consists of the following elements.

a. A blackbody source and chopper.

b. A monochromator using an in-line, off axis, Ebert configuration and interchangable gratings covering the range 2.5-16 microns. The exit of the monochromator is a 100 micron diameter pinhole.

c. A flat, gold coated, mirror tilted at 44 degrees, to reflect radiation from the pinhole to the $\mathrm{f} / 15$ scanning mirror.

d. An $\mathbf{f} / 15$ mirror, radius of curvature $1314 \mathrm{~mm}$, tilted at 1 degree. This mimics the ISO telescope secondary mirror and can tipped independently in two axes to scan the image of the pinhole across the Fabry mirror and hence, via the lens under test, across the detector array.

e. The cryostat which includes an ISOCAM Fabry mirror, a filter wheel, a lens mechanism and a detector. The lens mechanism holds a single lens in its ISOCAM

Y. Kondo (ed.), Observatories in Earth Orbit and Beyond, 497-499.

(C) 1990 Kluwer Academic Publishers. Printed in The Netherlands. 


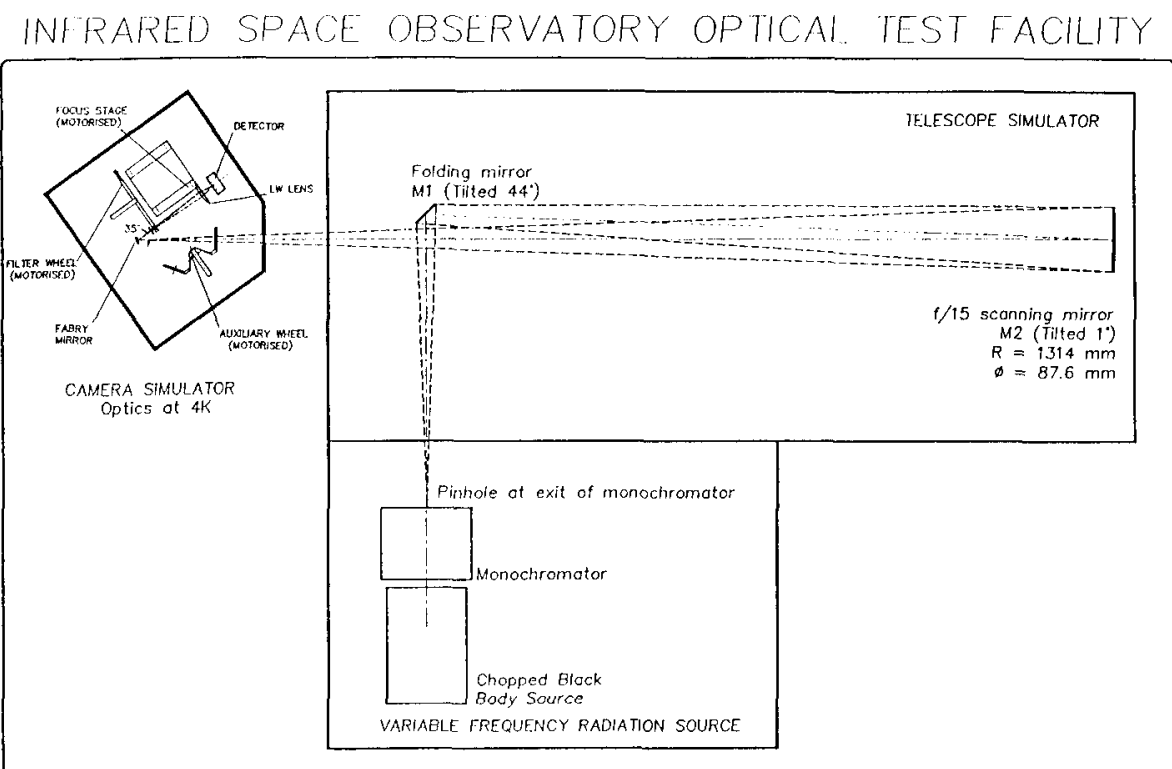

Fig. 1. Schematic Drawing of the ROE ISOCAM test facility.

mount and can be moved axially with a precision of 1 micron using a stepper motor located outsid e the cryostat.

In place of the detector array planned for ISOCAM, the test facility uses five individual SiGa elements mounted at the centre and edges of a simulated array. Each detector is fitted with a mask 60 microns in diameter.

The entire system is operated via an HP9816 mini computer which controls the stepper motors that move the lens, turn the filter wheel and scan the f/15 mirror. The HP9816 is also used to interrogate the detectors and store test data. An automated test se quence consisting of measurements over a range of different focus positions and filters can be run unattended overnight.

\section{Test Procedures}

By scanning the image of the monochromator exit pinhole across a detector and recording the signal at a number of mirror positions it is possible to reconstruct a beam profile, i.e. scanning mirror position versus signal received. This is a convolution of the object size, the point spread function of the lens and detector mask diameter. Since the size of the exit pinhole and the detector mask are known it is possible to extract the Point Spread Function (PSF) from the beam profile. By repeating the process at a number of different lens positions it is possible to determine the position of best focus for the lens, ie the Back Focal Length (BFL). The use of different detectors allows tests to be made both on and off axis. The derived BFL and PSF, at selected wavelengths, can then be compared with the theoretical values from the Code $\mathrm{V}$ package. The experimental results are used in the assembly 
TABLE I

Comparison of ISOCAM 6 arc sec/pixel silicon lenses.

\begin{tabular}{lllll}
\hline & QM & FM & FS & Theory \\
\hline PSF $(\mu \mathrm{m})$ & $43 \pm 6$ & $45 \pm 5$ & $46 \pm 5$ & $45($ at $3.5 \mu \mathrm{m})$ \\
PSF $(\mu \mathrm{m})$ & $59 \pm 6$ & $57 \pm 5$ & $62 \pm 5$ & $60($ at $4.5 \mu \mathrm{m})$ \\
BFL $(\mathrm{mm})$ & $32.90 \pm 0.3$ & $32.86 \pm 0.4$ & $32.84 \pm 0.4$ & $32.6 \pm 0.2($ at $3.5 \mu \mathrm{m})$ \\
BFL $(\mathrm{mm})$ & $32.98 \pm 0.35$ & $32.93 \pm 0.35$ & $32.91 \pm 0.35$ & $32.8 \pm 0.2($ at $4.5 \mu \mathrm{m})$
\end{tabular}

of the camera; correct positioning of the lens is critical since the camera has no focussing capability once assembled.

Filter testing is done by placing an appropriate filter in the beam and scanning the monocromator over a range of wavelengths which include the nominal filter passband. Comparison with a similar scan made without the filter in the beam enables the filter transmission to be found. Although this method is subject to complicating factors such as baseline drift between scans and the defocussing caused by inserting a filter into a converging optical beam it has proved satisfactory in practice.

\section{Results}

At this writing ROE have tested a total of eighteen ISOCAM lenses, comprising 8 for the Qualification Model, 4 for the Flight Model and 6 Flight Spares. It has been found that agreement between theory and experiment is good and that the BFL and PSF deriv ed for nominally identical lenses, ie those of a given pixel size for the QM,FM and FS, are in very close agreement with each other. This is notable because for contractural reasons the QM optics were machined by a different company to the FM and FS optics. An example is given in Table $\mathrm{I}$.

A complete set of ISOCAM interference filters (comprising 21 filters) has also been tested. The transmissions and passbands for the entire set were found to be satisfactory and in agreement with manufacturer's data taken at $77 \mathrm{~K}$ and extrapolated to $4 \mathrm{~K}$.

\section{Conclusions}

The ROE test facility has been used successfully to demonstrate that optics procured for ISOCAM are within specification and to supply values of BFL required in the assembly of the camera. The test facility is also available to provide experimental data in support of testing required during integration of the QM and FM cameras. 